\title{
Ethanol Withdrawal Drives Anxiety-Related Behaviors by Reducing M-type Potassium Channel Activity in the Lateral Habenula
}

\author{
Seungwoo Kang ${ }^{1,2}$, Jing $\mathrm{Li}^{1,2}$, Wanhong Zuo ${ }^{1,2}$, Rao Fu ${ }^{1,2}$, Danielle Gregor ${ }^{1,2}$, Kresimir Krnjevic ${ }^{3}$, \\ Alex Bekker' and Jiang-Hong Ye*,1,2 \\ 'Department of Anesthesiology, Rutgers, The State University of New Jersey, New Jersey Medical School, Newark, NJ, USA; ${ }^{2}$ Department of \\ Pharmacology, Physiology, and Neuroscience, Rutgers, The State University of New Jersey, New Jersey Medical School, Newark, NJ, USA; \\ ${ }^{3}$ Department of Physiology, McGill University, Montreal, Canada
}

\begin{abstract}
Alcohol use disorders (AUDs) and anxiety disorders (ADs) are often seen concurrently, but their underlying cellular basis is unclear. For unclear reasons, the lateral habenula (LHb), a key brain region involved in the pathophysiology of ADs, becomes hyperactive after ethanol withdrawal. M-type $\mathrm{K}^{+}$channels (M-channels), important regulators of neuronal activity, are abundant in the LHb, yet little is known about their role in AUDs and associated ADs. We report here that in rats at $24 \mathrm{~h}$ withdrawal from systemic ethanol administration (either by intraperitoneal injection, $2 \mathrm{~g} / \mathrm{kg}$, twice/day, for 7 days; or intermittent drinking $20 \%$ ethanol in a two-bottle free choice protocol for 8 weeks), the basal firing rate and the excitability of LHb neurons in brain slices was higher, whereas the amplitude of medium afterhyperpolarization and $\mathrm{M}$-type $\mathrm{K}^{+}$currents were smaller, when compared to ethanol naive rats. Concordantly, $\mathrm{M}$-channel blocker (XE99I)-induced increase in the spontaneous firing rate in LHb neurons was smaller. The protein expression of M-channel subunits, KCNQ2/3 in the LHb was also smaller. Moreover, anxiety levels (tested in open field, marble burying, and elevated plus maze) were higher, which were alleviated by LHb inhibition either chemogenetically or by local infusion of the M-channel opener, retigabine. Intra-LHb infusion of retigabine also reduced ethanol consumption and preference. These findings reveal an important role of LHb M-channels in the expression of AUDs and ADs, and suggest that the M-channels could be a potential therapeutic target for alcoholics.

Neuropsychopharmacology (2017) 42, I813-1824; doi:I0.1038/npp.2017.68; published online 10 May 2017
\end{abstract}

\section{INTRODUCTION}

Anxiety disorders (ADs), common symptoms of alcohol withdrawal, are important factors in the negative reinforcement leading to relapse (Driessen et al, 2001; Sinha, 2001; Wright et al, 1990). There is much interest in brain regions that drive anxiety in alcoholics. Most studies on alcoholrelated anxiety in animal models focus on the amygdaloid structures (Gilpin et al, 2015; McBride, 2002; Pandey et al, 2003, 2006). Multidisciplinary work collectively suggests that the central amygdala is an integrative hub for ADs and alcohol use disorder (AUDs) (Gilpin et al, 2015). However, the cellular basis underlying the comorbidity of $\mathrm{ADs}$ and AUDs has not been completely uncovered. The lateral habenula (LHb) has received increasing attention recently because of its pivotal role in aversive behaviors (Proulx et al, 2014). Besides the well-known functions of the LHb such as

*Correspondence: Professor J-H Ye, Department of Anesthesiology, Rutgers, The State University of New Jersey, New Jersey Medical School, 185 South Orange Avenue, Newark, NJ, 07I03, USA, Tel: 973972 1866, Fax: 973972 0582, E-mail: ye@njms.rutgers.edu Received 18 July 2016; revised 17 March 2017; accepted 26 March 2017; accepted article preview online 7 April 2017 the regulation of sleep and maternal behavior (Hikosaka, 2010), recent studies have shown that the LHb also acts as an important part in the reward circuit by providing 'negative value' signals to neuromodulator systems, particularly the dopaminergic and serotonergic systems (Proulx et al, 2014). The LHb relays information from the limbic forebrain to monoaminergic centers (Baldwin et al, 2011), controlling mood and emotions. Therefore, LHb disturbances have been implicated in the pathogenesis of psychiatric disorders such as depression and anxiety (Li et al, 2013; Zhao et al, 2015). Indeed, LHb hyperactivity is anxiogenic (Pobbe and Zangrossi, 2008). However, a role for the LHb in ADs associated with AUDs has not been demonstrated.

Since the transmission of neuronal signals relies critically on ion channels (Waxman and Zamponi, 2014), dysregulation of channel function in response to drug abuse may result in neuropathology. Over the past few decades, various $\mathrm{K}^{+}$ channels have been identified as major sites of regulation in the homeostatic plasticity of intrinsic membrane excitability (Misonou, 2010). M-type voltage-gated $\mathrm{K}^{+}$channels (M-channels) have received particular attention because they tend to repolarize neurons and thus prevent repetitive firing (Delmas and Brown, 2005; Vervaeke et al, 2006). 
Accumulating evidence suggests that M-channels could be a target of alcohol's actions on neuronal function and behavior (Cavaliere et al, 2012; Knapp et al, 2014; Koyama et al, 2007; McGuier et al, 2015; Moore et al, 1990). A previous histological study had shown that the M-channel subunit KCNQ2 is abundantly expressed in the LHb (Castro et al, 2001). We therefore hypothesized that a reduction of LHb $\mathrm{M}$-channel function may contribute to the anxiogenic effect of alcohol withdrawal, and tested this idea in experiments on rats, by combining electrophysiological, biochemical, chemogenetic, and behavioral approaches.

We found that during ethanol withdrawal, anxiety levels of rats and the activity of LHb neurons were increased, and M-channel expression was reduced. The elevated anxiety levels could be attenuated by selective inhibition of the LHb neurons with designer receptors exclusively activated by designer drugs (DREADDs) (Armbruster et al, 2007; Smith et al, 2016) or by the M-channel opener, retigabine. These findings suggest that $\mathrm{M}$-channels play an important role in the pathology of ADs after alcohol withdrawal.

\section{MATERIALS AND METHODS}

\section{In Vivo Systemic Administration of Ethanol}

Male Sprague Dawley (SD) rats (4-5-week-old) were given intraperitoneal injections (i.p.) of ethanol $(2 \mathrm{~g} / \mathrm{kg}$ in $20 \% \mathrm{v} / \mathrm{v}$ ), or an equivalent volume of saline twice a day for 7 days. Anxiety-related behaviors or neuronal properties were evaluated $24 \mathrm{~h}$ after the last injection. We selected this time point based on a previous observation of maximal anxiety-like behaviors in rats at $24 \mathrm{~h}$ of abstinence from repeated ethanol exposure (Gibula-Bruzda et al, 2015). Each behavior was tested in a separate group.

\section{Intermittent Access to 20\% Ethanol Two-Bottle Free Choice Drinking (IA2BC)}

We measured ethanol intake in the Long Evans rat, a strain commonly employed for voluntary ethanol consumption, in the IA2BC drinking paradigm (Li et al, 2011b; Simms et al, 2008; Wise, 1973). Detailed IA2BC paradigm is provided in Supplementary Materials and Methods.

\section{Stereotaxic Surgery and Microinjections}

Stereotaxic surgery and histological verification were performed as described (Li et al, 2016; Zuo et al, 2015). The details are provided in Supplementary Materials and Methods.

Intra-LHb Chemogenetic Virus Injection and Clozapinen-oxide (CNO) Treatment

We introduced the engineered human muscarinic receptor, either the inhibitory hm4D or the excitatory hm3Dq by injecting AAV5-CaMKIIa-hm4D-mCherry, AAV5-CaMKIIa-hm3Dq-mCherry, or control AAV5-CaMKIIa-eGFP (titers of $10^{12}-10^{13} \mathrm{vg} / \mathrm{ml}$, UNC Vector Core, Chapel Hill, $\mathrm{NC})$ bilaterally into the $\mathrm{LHb}(\mathrm{AP}-3.4 \mathrm{~mm}, \mathrm{ML} \pm 0.73 \mathrm{~mm}$, DV $-4.8 \mathrm{~mm}$ ) of SD rats at P32-35 weighing 100-120 g. A volume of $350 \mathrm{nl}$ per side was delivered at a rate of
$70 \mathrm{nl} \mathrm{min}{ }^{-1}$. Ethanol injections were started 2-weeks later. At $24 \mathrm{~h}$ after the last ethanol injection, $\mathrm{CNO}(5 \mathrm{mg} / \mathrm{kg}$, dissolved in $0.5 \%$ DMSO v/v saline, i.p.) was given $30 \mathrm{~min}$ before the behavioral test (Smith et al, 2016). Ethanol naive rats were injected with saline and received the same handling as ethanol-treated rats.

\section{Measurement of Anxiety-like Behaviors}

The anxiety-like behaviors were measured by elevated plus maze test (EPM), Marble burying test (MBT), and Open field test (OPT). The detailed conditions of each test are described in Supplementary Materials and Methods.

\section{Brain Slice Preparation and Electrophysiology}

The brain slices preparation and electrophysiology were performed according to previously described criteria (Zuo et al, 2015)(Supplementary Materials and Methods).

\section{Western Blotting, Immunofluorescence, and Antibodies}

The procedures of western blotting and immunofluorescence and all antibodies used in the present study are described in Supplementary Materials and Methods.

\section{Measurements of Blood Ethanol Concentration}

Blood samples were acquired from the lateral tail vein of rats $2 \mathrm{~h}$ after $\mathrm{LHb}$ infusion of aCSF or retigabine and the ethanol concentration was measured as described (Carnicella et al, 2009; Li et al, 2011b; Simms et al, 2008).

\section{Intermittent Access to Sucrose Using a Two-Bottle Choice Drinking Protocol}

A separate group of rats implanted with cannulae in the LHb, were trained to drink sucrose under intermittent access to $2 \%$ sucrose by the two-bottle choice procedure, 7 days after the surgery, as described (Li et al, 2011b).

\section{Drugs}

We purchased common salts and apamin from Sigma Aldrich (St. Louis, MO, USA), retigabine from Alomone (Jerusalem, Israel), XE991 from Tocris (Bristol, UK), and ethanol from Pharmco Products Inc (Brookfield, CT). CNO was from NIDA Drug supply program (NIH, Bethesda, MD).

\section{Data Analysis and Statistics}

We measured the mean frequency of spontaneous firing over the last 3 -min of 5 -min periods of recording and calculated drug-induced changes by normalizing the data to the preceding 3-min of baseline firing. Statistical analyses were performed using Prism (Graphpad, La Jolla, CA). All compiled data are presented as mean \pm SEM. Statistical significance was assessed using paired or unpaired $t$-tests, and one- or two-way ANOVA with post hoc multiplecomparisons, when appropriate. Values of $p<0.05$ were considered significant. 

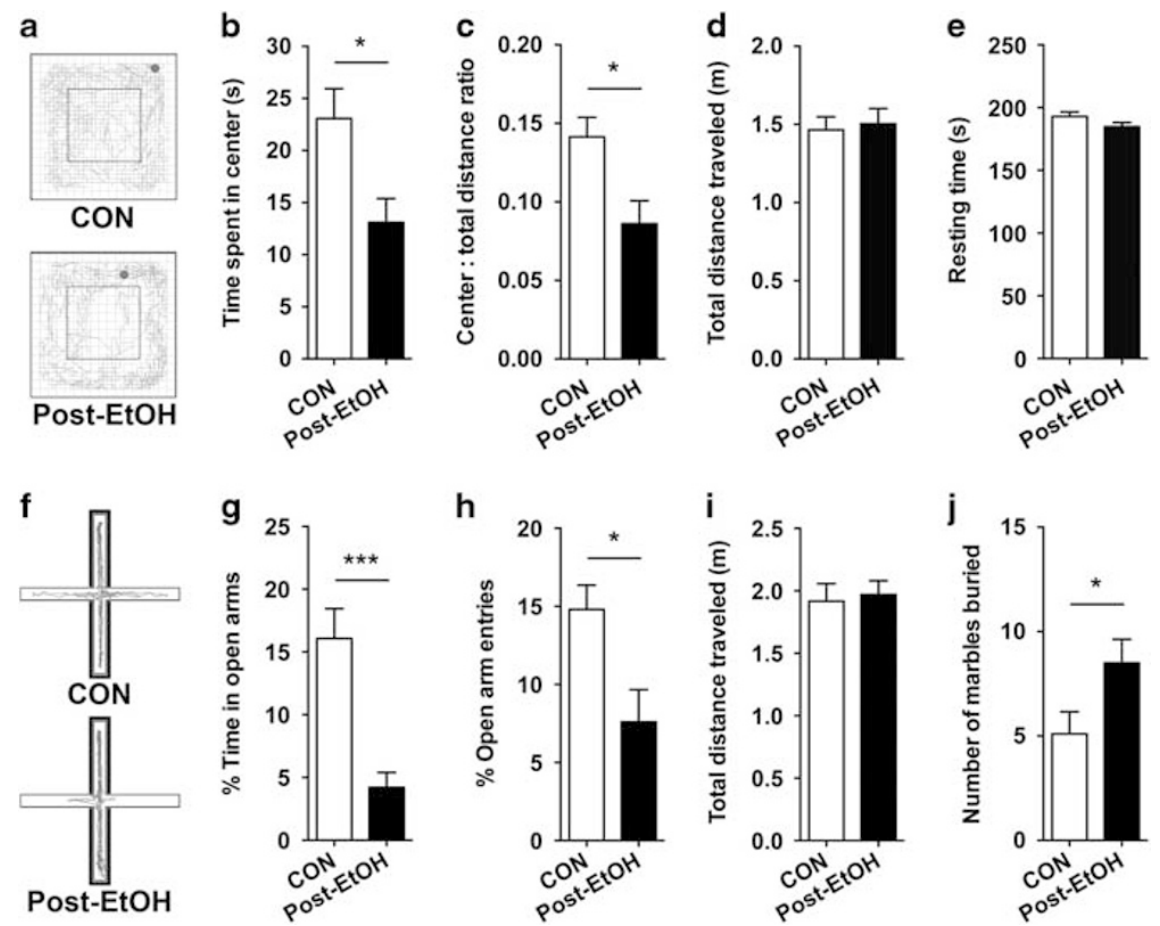

Figure I Anxiety-like behaviors in rats withdrawn from systemic administration of ethanol. (a-e) Open field data. (a) Representative traces show time spent in center of chamber by rats at $24 \mathrm{~h}$ withdrawal from repeated systemic ethanol administration (Post-EtOH) are shorter than that by ethanol-naive rats $(C O N)$. (b-e) Mean ( \pm SEM) data $\left(\mathrm{N}_{\text {rat }}=\right.$ I0/group): (b) mean times spent in center (Unpaired $t$-test, $\left.t=2.7 \mid \mathrm{I}\right)$, (c) proportion of total distance travelled at center, (d) total distances traveled, and (e) resting time. Unpaired t-test: $* p<0.05$, $* * * 0.01$. ( $f-j$ ) Elevated plus maze data: ( $f$ ) Representative traces show Post-EtOH rats spend much less time in open arms than do CON rats. (g-i) Summary data: (g) time spent in open arms; (h) entries into open arms, and (i) total distance traveled by $\mathrm{CON}$ and Post-EtOH rats. Unpaired t-test: $* 0<0.05 . \mathrm{N}_{\text {rat }}=10$ /group. (j) Number of marbles buried in 30-min marble-burying anxiety test. Unpaired t-test: $* p<0.05, * * * * 00.001$. $N_{\text {rat }}=10 /$ group.

\section{RESULTS}

\section{Anxiety-like Behaviors in Juvenile Rats after Discontinuing Ethanol Administration}

Anxiety-like behaviors were examined $24 \mathrm{~h}$ after the last injection of ethanol $(2 \mathrm{~g} / \mathrm{kg}$, i.p. twice per day for $7 \mathrm{~d}$, PostEtOH rat) or saline (CON rats). In the OPT, while the total distance traveled and resting time were similar (Figure 1a, d and e), the distance traveled and time spent in the center of the open field were significantly shorter for Post-EtOH rats than $\mathrm{CON}$ rats (Figure 1a-c), suggesting increased anxiety levels. Correspondingly, the time spent in the open arms and the entries into open arms of the EPM were significantly reduced for Post-EtOH rats compared to $\mathrm{CON}$ rats (Figure 1f-i). Likewise, Post-EtOH rats buried significantly more marbles than did CON rats in the MBT (Figure 1j).

\section{Increased Activity of LHb Neurons in Slices from Juvenile Rats after Discontinuing In Vivo Ethanol Administration}

To assess the role of the LHb in the anxiety-like behaviors seen after ethanol withdrawal, we first measured the spontaneous activity of neurons in the LHb, particularly those in the medial region (Figure 2a), where neurons project mainly to the dorsal raphe (Proulx et al, 2014), a brain area that controls generalized anxiety in rats (Sena et al, 2003; Spiacci et al, 2012). The basal firing rate of LHb neurons in slices from Post-EtOH rats was significantly higher than that of $\mathrm{CON}$ rats (Figure $2 \mathrm{a}$ and $\mathrm{b}$ ).

\section{Chemogenetic Inhibition of LHb Neurons Alleviates Anxiety-like Behaviors of Juvenile Rats}

To further assess the role of the LHb in anxiety-like behaviors after ethanol withdrawal, we recorded from $\mathrm{LHb}$ neurons in slices from rats infected three weeks earlier with either an AAV5 carrying hm4D inhibitory muscarinic receptors or eGFP control virus bilaterally in the $\mathrm{LHb}$ (Figure $2 \mathrm{c}$ and d). Bath application of CNO $(5 \mathrm{uM})$ significantly reduced the spontaneous firing of $\mathrm{LHb}$ neurons in slices infected with hm4D but not those infected with eGFP (Figure 2e and f). Importantly, systemic administration of CNO $(5 \mathrm{mg} / \mathrm{kg}$, i.p.) $24 \mathrm{~h}$ after the last injection of ethanol $(2 \mathrm{~g} / \mathrm{kg}$, i.p. twice per day for 7 days) significantly alleviated the anxiety-like behaviors in the EPM (Figure $2 \mathrm{~g}-\mathrm{j}$ ) and in the MBT of rats infected with hm4D but not in those infected with eGFP (Figure 2k). Rats injected with AAV-eGFP did not show any difference in baseline levels in the EPM and the MBT compared to those of CON rats without viral injection (effects of virus; \% time in open arms, $F_{1,46}=3.302, p=0.0757$; number of marbles buried, $\left.\mathrm{F}_{1,38}=1.886, p=0.1777\right)$, and both groups of rats showed anxiety-like behaviors after repeated ethanol injection, regardless of viral injection (effects of $\mathrm{EtOH}$; \% time in open arms, $\mathrm{F}_{1,46}=20.55, p<0.0001$; number of marbles buried, $F_{1,38}=16.49, p=0.0002$; Figures $1 \mathrm{~g}, \mathrm{j}$ and $2 \mathrm{~h}, \mathrm{k}$ ). 


\section{Chemogenetic Activation of LHb Neurons Initiates Anxiety-like Behaviors in Ethanol Naive Rats}

To ascertain the role of the LHb in the initiation of anxietylike behaviors, we mimicked the ethanol-withdrawn states of
LHb neurons by bilaterally transducing LHb neurons with AAV5 that carried the chemogenetic receptor, hm3Dq, under the control of the CaMKIIa promoter. Hm3Dq is a modified muscarinic receptor 3 that effectively induces firing of neurons in response to the ligand, CNO (Alexander et al, a

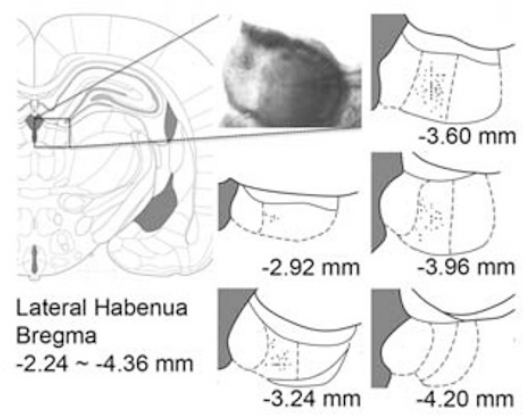

b

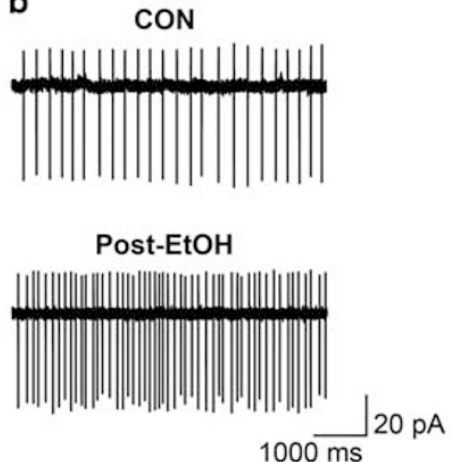

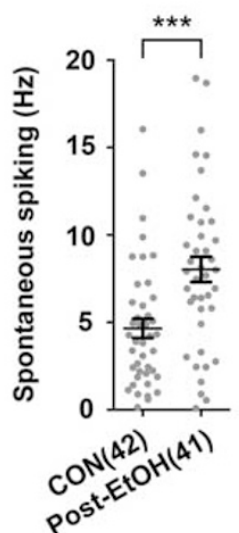

c

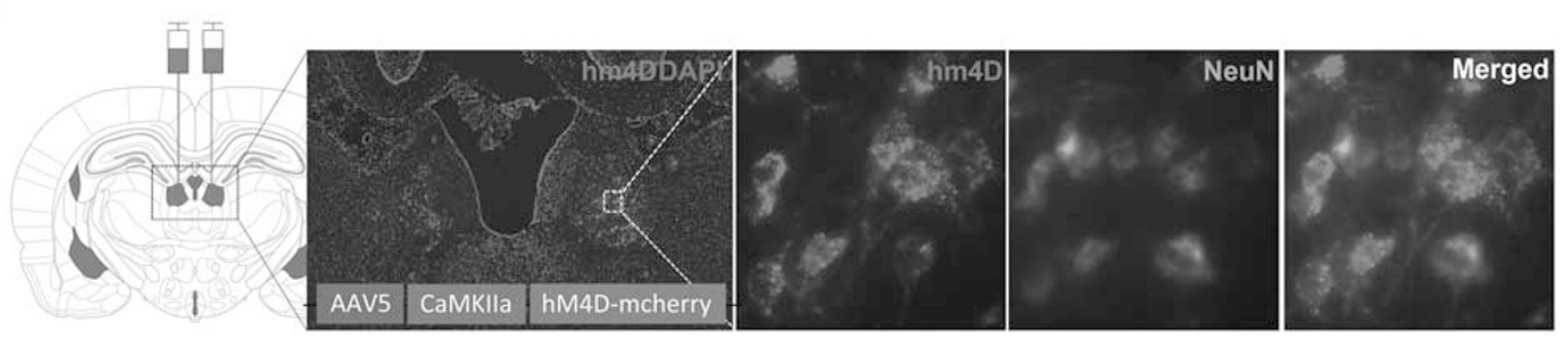

d

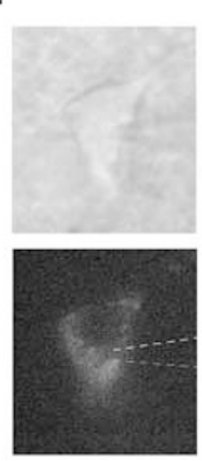

g

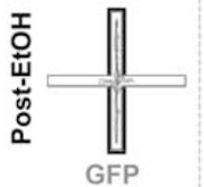

e1

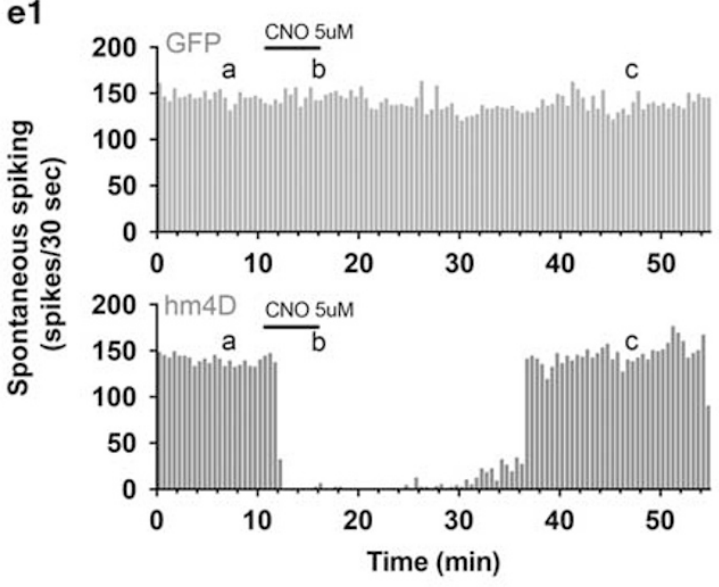

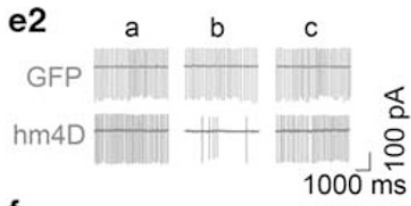

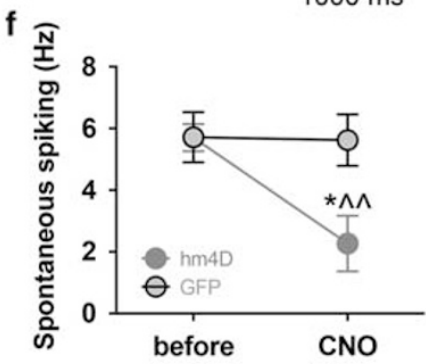<smiles></smiles>

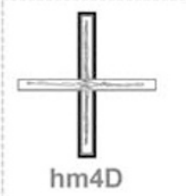

h

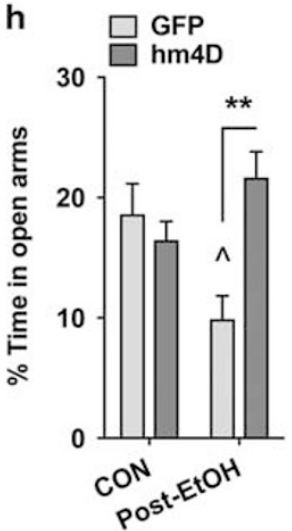

i

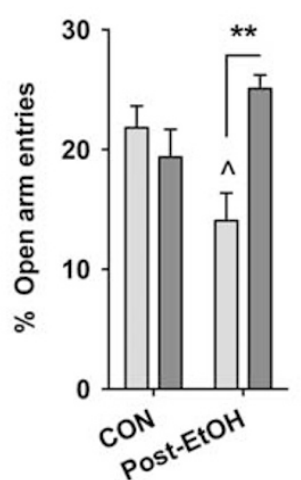

j

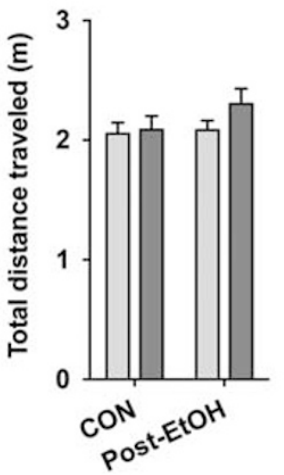

k

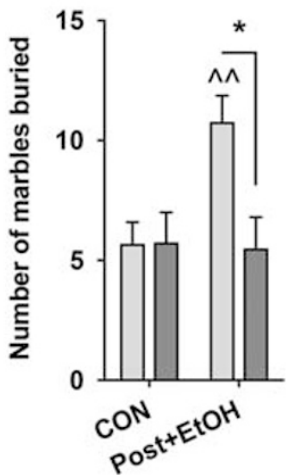


2009). As expected, bath application of CNO increased the spontaneous firing rate of the $\mathrm{LHb}$ neurons in slices from rats infected with hm3Dq but not those infected with eGFP (Supplementary Figure S1A and B). Moreover, activation of $\mathrm{LHb}$ neurons by systemic administration of $\mathrm{CNO}$ $(5 \mathrm{mg} / \mathrm{kg}$, i.p.) significantly decreased the time spent in the open arms of the EPM (Supplementary Figure S1C-F) and the number of buried marbles in the MBT (Supplementary Figure S1G).

\section{Changes in Intrinsic Properties of LHb Neurons in Slices from Juvenile Rats Withdrawn from Systemic Ethanol Exposure}

To investigate the mechanism of LHb hyperactivity in ethanol-withdrawn rats, we injected a series of incremental depolarizing current pulses into LHb neurons. Such currents evoked more firing in $\mathrm{LHb}$ neurons from Post-EtOH rats than those from CON rats (Figure $3 a-c$ ), suggesting that ethanol withdrawal increases the intrinsic excitability of LHb neurons.

Changes in action potentials may result from altering voltage-gated $\mathrm{Na}^{+}$and/or voltage-gated $\mathrm{K}^{+}$currents (Kourrich et al, 2015). Increased excitability could result from increased $\mathrm{Na}^{+}$channel function (Halter et al, 1995; Zhang et al, 1998). However, this is unlikely a major factor since action potential thresholds and peak amplitudes were similar in $\mathrm{LHb}$ neurons from CON and PostEtOH rats (Figure 3b, d, e and i). We therefore examined the possible contribution of $\mathrm{K}^{+}$channels. Resting membrane potentials (RMP) and action potential durations did not differ between LHb neurons from CON and Post-EtOH rats (Figure $3 b, f$ and $h$ ), suggesting no change in inwardly rectifying $\mathrm{K}^{+}$current. Conversely, close examination of the $\mathrm{K}^{+}$channel-mediated afterhyperpolarization revealed that whereas its earliest component, the fast after hyperpolarization (fAHP) was unchanged (Figure $3 \mathrm{j}$ ), the second one (medium afterhyperpolarization, mAHP) was significantly smaller in LHb neurons of Post-EtOH rats (Figure 3k), resulting in shorter inter-spike interval in Post-EtOH rats than in CON rats (Figure 3g).

\section{Involvement of M-current in Ethanol-Induced Adaptation of LHb Neurons of Juvenile Rats}

The mAHP is generated mainly by small conductance $\mathrm{Ca}^{2+}$-activated $\mathrm{K}^{+}$channels (SK channels) and M-type $\mathrm{K}^{+}$channels (M-channels) (Gu et al, 2005; Kang et al, 2014). Since KCNQ2, a subtype of the M-channel, is abundant in the LHb (Castro et al, 2001), we tested the effects of XE991, a KCNQ-channel blocker. As expected, XE991 $(20 \mu \mathrm{M})$ substantially accelerated the firing of $\mathrm{LHb}$ neurons in slices from CON rats, confirming the existence of functional M-channels (Figure 31 and $\mathrm{n}$ ). Importantly, XE991's effect was significantly weaker in the LHb neurons of Post-EtOH rats than in $\mathrm{LHb}$ neurons of CON rats, consistent with a reduction of $\mathrm{M}$-channel function in $\mathrm{LHb}$ neurons of Post-EtOH rats (Figure 3l, $\mathrm{n}$ and p).

The SK type of $\mathrm{K}^{+}$channel that contributes to the mAHP is also present in the LHb although at low levels (Pedarzani et al, 2000; Vielhaber et al, 2004). Bath application of the SK channel blocker, apamin $(100 \mathrm{nM})$, significantly accelerated firing of $\mathrm{LHb}$ neurons (Figure $3 \mathrm{~m}$ and o) similarly in both the $\mathrm{CON}$ and Post-EtOH rats (Figure 3p). These results suggest that the hyperactivity of $\mathrm{LHb}$ neurons in Post-EtOH rats is mediated by the reduction of M-channel function. This possibility is further supported by the observation that although XE991 $(20 \mu \mathrm{M})$ significantly reduced mAHP (Supplementary Figure S2A and E) and increased the number of spikes evoked by a depolarizing pulse in LHb neurons from CON rats (Supplementary Figure S2A and C), it had no significant effect on LHb neurons from Post-EtOH rats (Supplementary Figure S2B, D and E).

To measure M-current directly (Supplementary Figure S3), we injected $10 \mathrm{mV}$ hyperpolarizing steps from -30 to $-60 \mathrm{mV}$ at a holding potential of $-20 \mathrm{mV}$. The slow inward relaxation-reflecting the closing of the voltage-dependent M-current-was largest during the step to $-40 \mathrm{mV}$ (left panel in Figure 4a and open circles in Figure 4b). The much smaller inward relaxations in LHb neurons of Post-EtOH rats (Figure 4a and b) indicate a correspondingly weaker M-channel activity. Since the total cell capacitance was similar for cells from both groups of rats, the difference in M-current is unlikely to be due to a difference in cell size (Figure 4c).

Figure 2 Withdrawal from systemic ethanol administration increases activity of LHb neurons in brain slices and chemogenetic inhibition of the LHb attenuates anxiety-like behaviors. (a) Schematic of location of recorded neurons. (b) Representative traces and summary data show increased spontaneous firing of $\mathrm{LHb}$ neurons after ethanol withdrawal. Unpaired t-test: ***** $<0.00 \mathrm{I}$. Numbers of neurons are indicated. (c) hm4D-mcherry expression in LHb neurons after viral vector injection and immunofluorescence of the neuronal marker NeuN. Strong signal of hm4DmCherry is overlapped with NeuN. (d) CCD camera captured IR (upper) and ET-DSRed filtered fluorescence (bottom) image of the LHb neuron after viral injection for loose cell-attached patchclamping recording. (e, f) Bath applied CNO $(5 \mu \mathrm{M})$ sharply reduced the firing rate of LHb neurons infected with AAV-CaMKIla-hm4D-mCherry viruses (hm4D), but not those with control AAV5-CaMKIla-eGFP (GFP). Two-way ANOVA, for Drug treatment $F_{1,14}=20.14, p=0.0005$, for interaction $F_{1,14}=18.03, p=0.0008$, Bonferroni's post hoc test: * $p<0.05$ vs hmD4 before, ${ }^{\wedge} \wedge p<0.0$ I vs GFP CNO. $N_{\text {cell }}=8 /$ group. (g-k) Elevated plus maze data: (g) representative traces show that after systemic CNO injection $(5 \mathrm{mg} / \mathrm{kg}$, i.p.), Post-EtOH rats but not the CON rats, infected with hm4D spend more time in open arms than those infected with eGFP. (h-i) Summary of times spent in open arms (for GFP/hm4D expression $F_{1,46}=4.184$, $p=0.0466$, for interaction $F_{1,46}=8.795, p=0.0048$, post hoc test: ${ }^{\wedge} p<0.05$ vs CON GFP, $* * *<0.01$ vs Post-EtOH GFP, H), entries into open arms (for GFP/hm4D expression $F_{1,46}=4.443, p=0.0405$, for interaction $F_{1,46}=10.93, p=0.0018$, post hoc test: ${ }^{\wedge} p<0.05$ vs CON GFP, $* * * 0.0$ l vs Post-EtOH GFP, I) and total distance traveled (for GFP/hm4D expression $\mathrm{F}_{1,46}=1.679, p=0.2015$, for interaction $\mathrm{F}_{1,46}=0.90 \mathrm{I} \mathrm{I}, p=0.3474$, post hoc test: all $p>0.05$, J) by rats infected with GFP (grey bars) or hm4D (dark bars), as revealed by two-way ANOVA, Bonferroni's post hoc test. $\mathrm{N}_{\text {rat }}=16(\mathrm{CON} \mathrm{GFP}),=8(\mathrm{CON}$ hm4D), $=14(\mathrm{Post}-\mathrm{EtOH}$ GFP), =12(Post-EtOH hm4D). ( $\mathrm{k}$ ) In 30-min MBTs performed after CNO injections, substantially fewer marbles were buried by hm4D-infected rats than by eGFP-infected rats during ethanol withdrawal. Two-way ANOVA, for drug treatment $F_{1,34}=5.37 \mathrm{I}, p=0.0266$, for interaction $F_{1,34}=5.6 \mathrm{II}, p=0.0237$, Bonferroni's post hoc test: ${ }^{\wedge} p<0.01$ vs CON GFP, ${ }^{*} p<0.05$ vs Post-EtOH GFP. $N_{\text {rat }}=13(\mathrm{CON}$ GFP), 8(CON hm4D), =9(Post-EtOH GFP), $=8($ Post$\mathrm{EtOH}$ hm4D). 
Down-Regulation of M-Channel Expression in the LHb of Ethanol-Withdrawn Juvenile Rats

Previous studies have identified that the heterotetramer channel complex KCNQ2/3 belonging to KCNQ family (Kv7) is the main molecular correlates of the native M-current (Shah et al, 2002; Wang et al, 1998) and have shown the prominent subcellular localization at somata, axon initial segments, and the nodes of KCNQ2 and KCNQ3 subunits (Klinger et al, 2011; Trimmer, 2015). The
M-channel subunits KCNQ2 (Castro et al, 2001) and KCNQ3 are abundantly expressed in the LHb (Supplementary Figure S4). Specifically, the immunoreactivities for KCNQ2 and KCNQ3 were observed in the somata of glutamatergic neurons in the $\mathrm{LHb}$ and appeared more pronounced in the membrane. KCNQ gene expression is correlated with M-current density and neuronal excitability (Mucha et al, 2010). To determine whether M-channel expression in the $\mathrm{LHb}$ is changed by withdrawal from chronic ethanol exposure, we quantified M-channel a

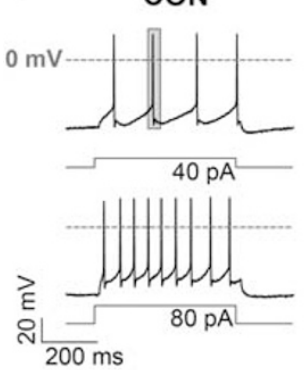

Post-EtOH
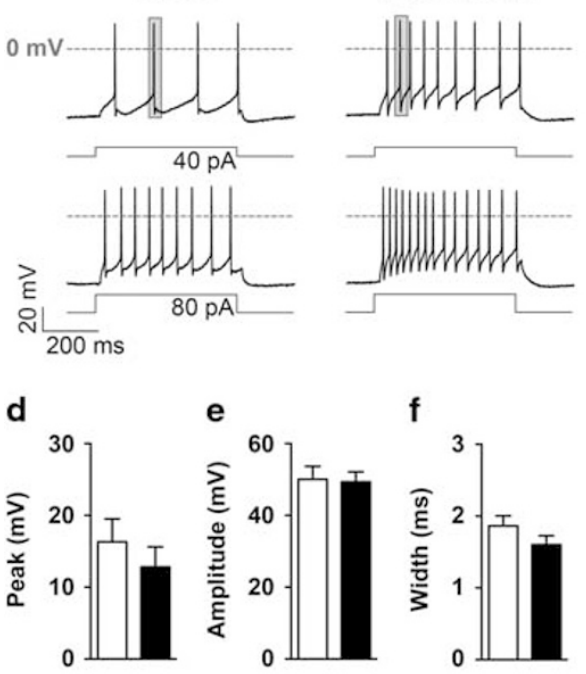

f

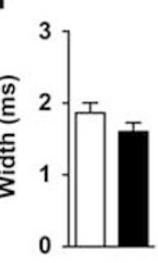

g

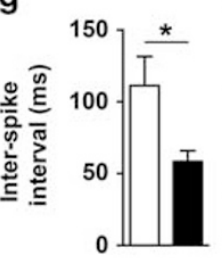

h

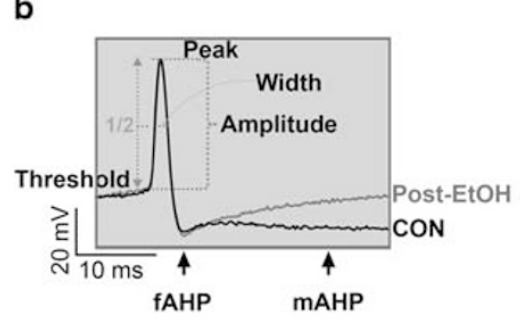

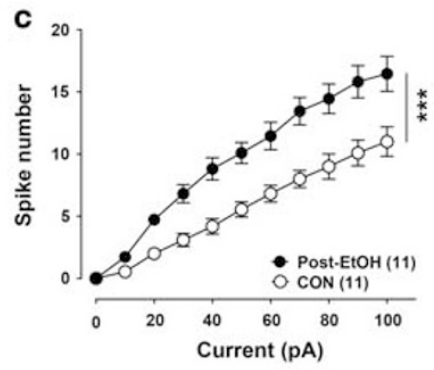

I
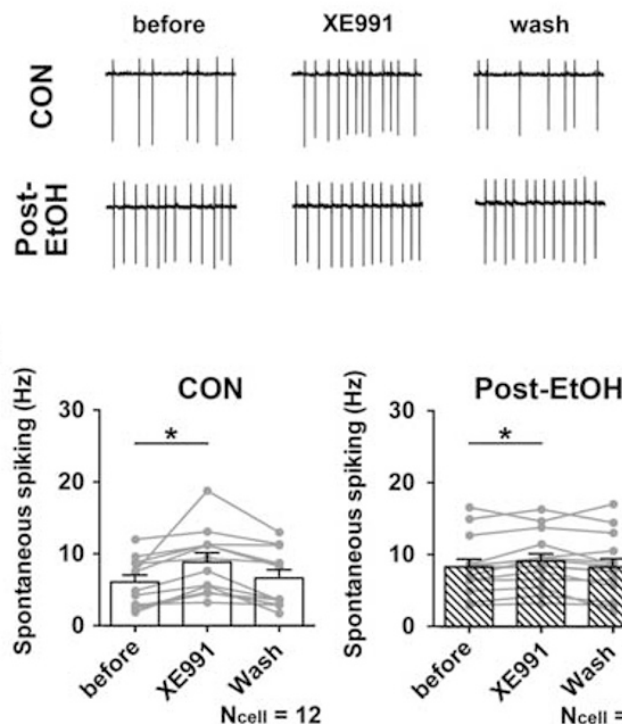

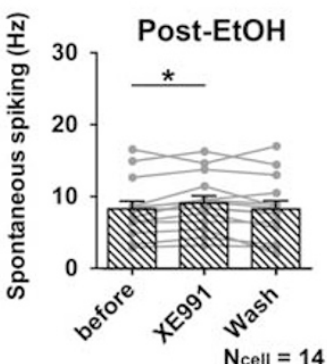

p

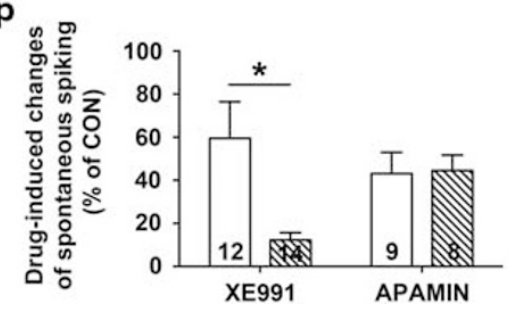

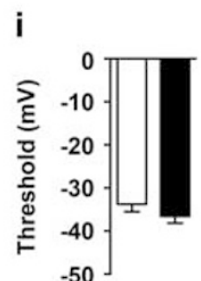

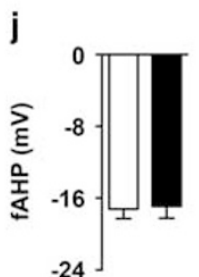

k

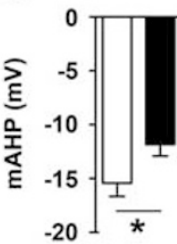

$\square$ CON
$-24$

Post-EtOH Ncell = 11/group
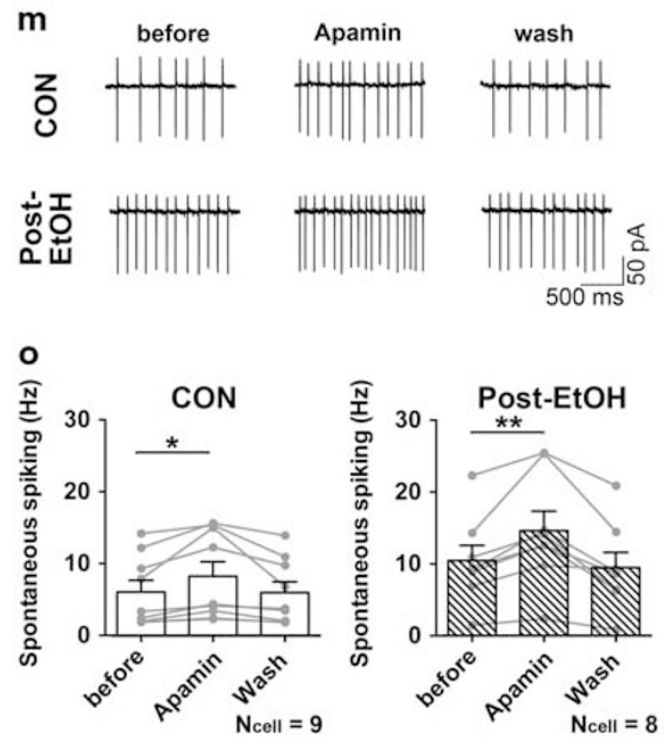

$\square$ CON

Most-EtOH 
expression in the LHb in Western blots. Both KCNQ2 and KCNQ3 subunits in $\mathrm{LHb}$ were significantly reduced in tissue from Post-EtOH compared to tissue from CON rats (Figure $4 \mathrm{~d}$ and $\mathrm{e}$ ). These results suggest that the observed smaller $\mathrm{M}$-currents could result from the lower expression of KCNQ2 and KCNQ3 in the LHb after ethanol withdrawal.

\section{Activation of LHb M-Channels Reduces Anxiety-like Behaviors in Ethanol-Withdrawn Rats}

To determine whether LHb M-channels contribute to the anxiety-like behaviors seen after ethanol withdrawal, we first examined the effect of the M-channel activator, retigabine, on LHb neuronal activity. As expected, bath application of retigabine dose-dependently reduced the spontaneous firing of $\mathrm{LHb}$ neurons in slices from both $\mathrm{CON}$ rats and Post-EtOH rats; and the reduction was significantly weaker in LHb neurons from Post-EtOH rats (Figure 5a and b), although retigabine at higher doses $(10$ and $30 \mu \mathrm{M})$ substantially decreased LHb activities of both groups of rats. Specifically, retigabine $(30 \mu \mathrm{M})$ sharply decreased $\mathrm{LHb}$ firing in slices from both $\mathrm{CON}$ (to $4.8 \pm 2.5 \%$ of baseline, $n=5$, paired $t$-test, $t=37.57, p<0.0001$ ) and Post-EtOH rats (to $10.6 \pm$ $8.0 \%$ of baseline, $n=5$, paired $t$-test, $t=11.16, p=0.0004$ ) (Figure 5b). Importantly, intra-LHb infusion of retigabine at
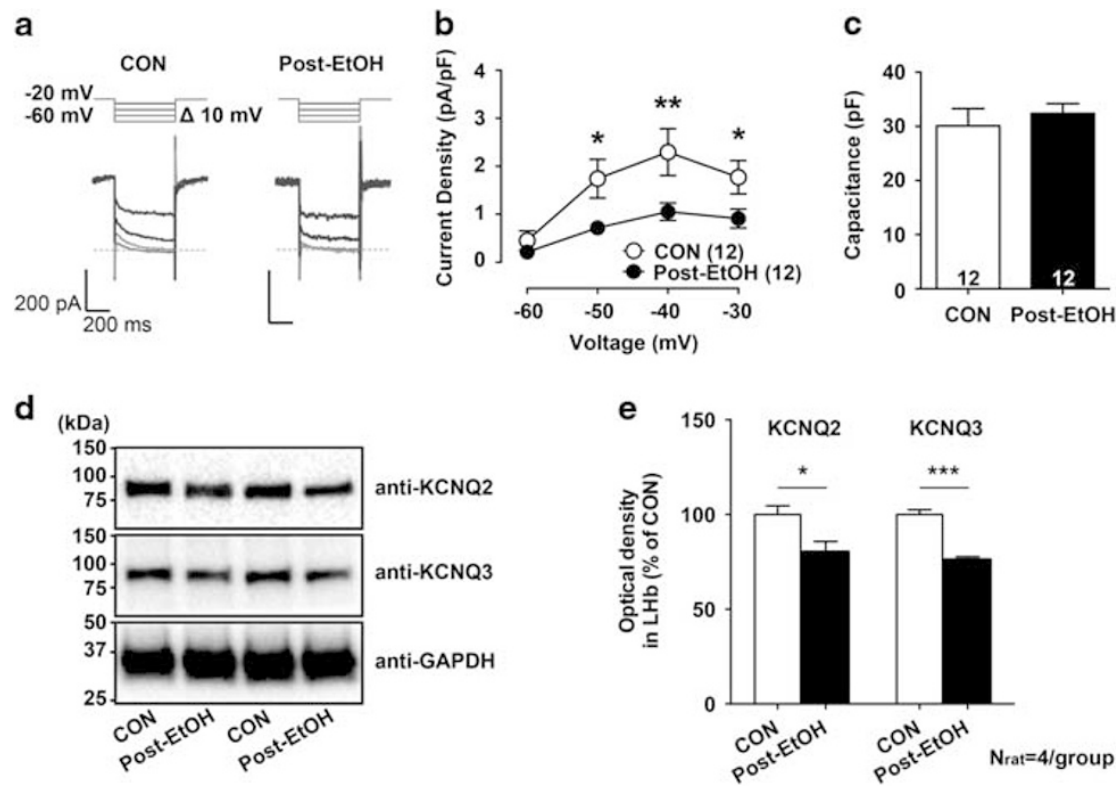

Figure 4 Reduction of M-current and expression of M-channels in LHb neurons in ethanol-withdrawn rats. (a) Sample traces show reduced inward current relaxations in neurons from ethanol-withdrawn rats $(\mathrm{Post}-\mathrm{EtOH})$ compared to neurons from ethanol-naive rats (CON). At a holding potential of $-20 \mathrm{mV}$, ongoing M-currents were deactivated by 500 -ms repolarizing steps from -30 to $-60 \mathrm{mV}$. (b) Density of M-currents is much lower in Post-EtOH rats than in CON rats. Two-way ANOVA, for $\mathrm{CON} / \mathrm{EtOH}$ treatment, $F_{1.22}=5.705, p=0.025$, for interaction, $F_{3,66}=3.619$, $p=0.017$, Bonferroni's post hoc test: ${ }^{*} p<0.05$, ** $p<0.01 . \mathrm{N}_{\text {cell }}=12 /$ group. (c) Pooled results show no difference in total cell capacitance between LHb neurons from ethanol-withdrawn and naive rats. Unpaired t-test, $t=0.6206, p=0.5413 . N_{\text {cell }}=12 /$ group. (d,e) Examples (d) and pooled results (e) of western blots show reduced KCNQ2 and $\mathrm{KCNQ} 3$ expression in the LHb from ethanol-withdrawn rats compared to CON rats. Note an even greater loss of KCNQ3 expression in the Post-EtOH rats. Unpaired $t$-test: for KCNQ2 $t=2.754$, * $p<0.05$, for KCNQ3 $t=8.287$, **** $<<0.00 \mathrm{I} . \mathrm{N}_{\text {rat }}=4$ /group.

Figure 3 Increased excitability and reduced M-channel blocker (XE99I)-induced increase in the firing rate LHb neurons in slices of rats withdrawn from systemic ethanol administration. (a) Current injections elicit significantly more spikes in $\mathrm{LHb}$ neurons of ethanol-withdrawn rats (Post-EtOH, right) than naive rats (CON, left). (b) Superimposed action potentials reveal loss of second phase of afterhyperpolarization (AHP) in Post-EtOH rats. (c) Number of spikes evoked over a wide range of current pulses was consistently higher in Post-EtOH rats than CON rats. Two-way ANOVA, for $\mathrm{CON} / \mathrm{EtOH} \mathrm{F}_{1,20}=18.58$, $p=0.0003$, for interaction $F_{10,200}=5.808, p<0.000 I$. $N_{\text {cell }}=1 \mid$ /group. (d-k) Electrical properties of LHb neurons in CON and Post-EtOH rats: (d) membrane potential at peak of spike (Unpaired $t$-test, $t=0.8259, p=0.4 \mathrm{I} 86$ ), (e) spike amplitude (Unpaired $t$-test, $t=0.158 \mathrm{I}, p=0.8760$ ), ( $f$ ) spike width (at the $1 / 2$ point from the peak to the threshold potential, Unpaired $t$-test, $t=1.406, p=0.1750)$, $(\mathrm{g})$ inter-spike time $($ Unpaired $t$-test, $t=2.442, p=0.0240)$, $(h)$ RMP (Unpaired $t$-test, $t=0.1922, p=0.8495)$, (i) spike threshold (Unpaired $t$-test, $t=1.191, p=0.2477$ ), (j) amplitude of fast AHPs (Unpaired $t$-test, $t=0.1663, p=0.8696)$, and $(k)$ medium AHPs (Unpaired $t$-test, $t=2.205, p=0.0393$ ) (With the threshold as a baseline, fAHP and mAHP were measured as the lowest point $25 \mathrm{~ms}$ and $20-40 \mathrm{~ms}$ after the peak of the action potential, respectively.). $* p<0.05 . \mathrm{N}_{\text {cell }}=1 \mathrm{I} /$ group. (I, n, p) Examples of firing recorded in cell-attached mode (I) and summary data $(n)$ show that the accelerated LHb firings induced by the M-channel blocker XE99I (20 HM) (One-way ANOVA, $F_{2,22}=10.5 \mathrm{I}, p=0.0006$, Bonferroni's post hoc test: $* * * * 0.000 \mathrm{I}$ ) is reduced in $\mathrm{LHb}$ neurons from rats $24 \mathrm{~h}$ after end of ethanol administration (Post-EtOH). Unpaired $t$-test: $t=2.947, p=0.007(p)$. ( $m, o, p)$ Examples of firing $(m)$ and summary data $(0)$ show that the SK channel blocker apamin $(I 00 \mathrm{nM})$ causes a similar acceleration of firing in LHb neurons from CON rats and Post-EtOH rats (for CON group, One-way ANOVA, $F_{2,16}=6.982, p=0.0066, \mathrm{Bonferroni}$ 's post hoc test: * $p<0.05 /$ for Post-EtOH group, One-way ANOVA, $F_{2,14}=14.44, p=0.0004$, Bonferroni's post hoc test: * $p<0.01 /$ for CON vs Post-EtOH, Unpaired $t$-test, $t=0.1098, p=0.9 \mid 40)$. ( $p)$ Histogram summarizing these results. Cell numbers are indicated. 
a

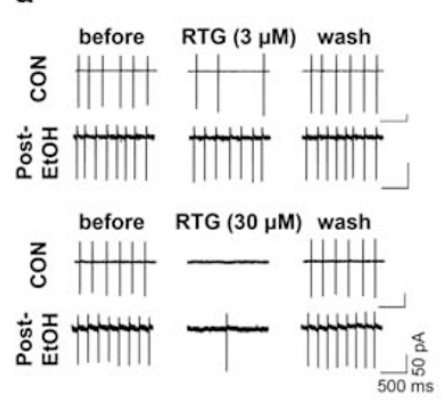

b

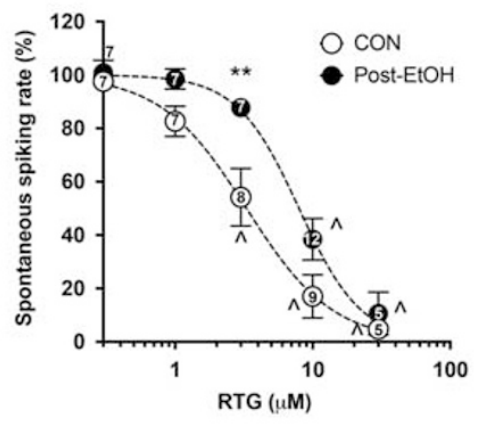

C

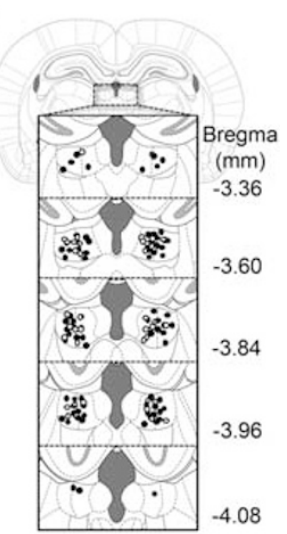

d

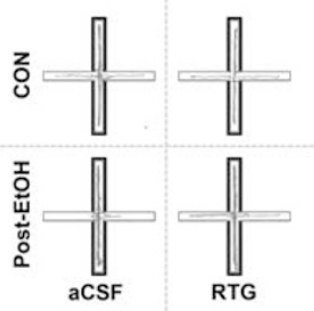

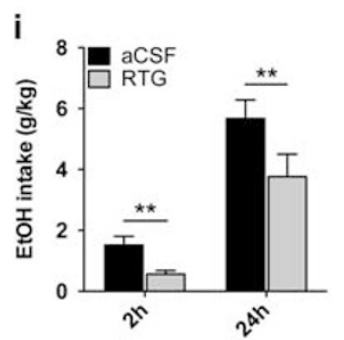

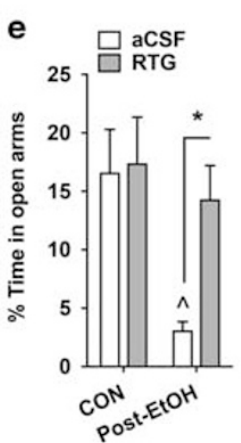

f

\section{$f$}

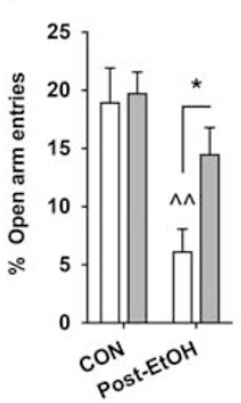

g
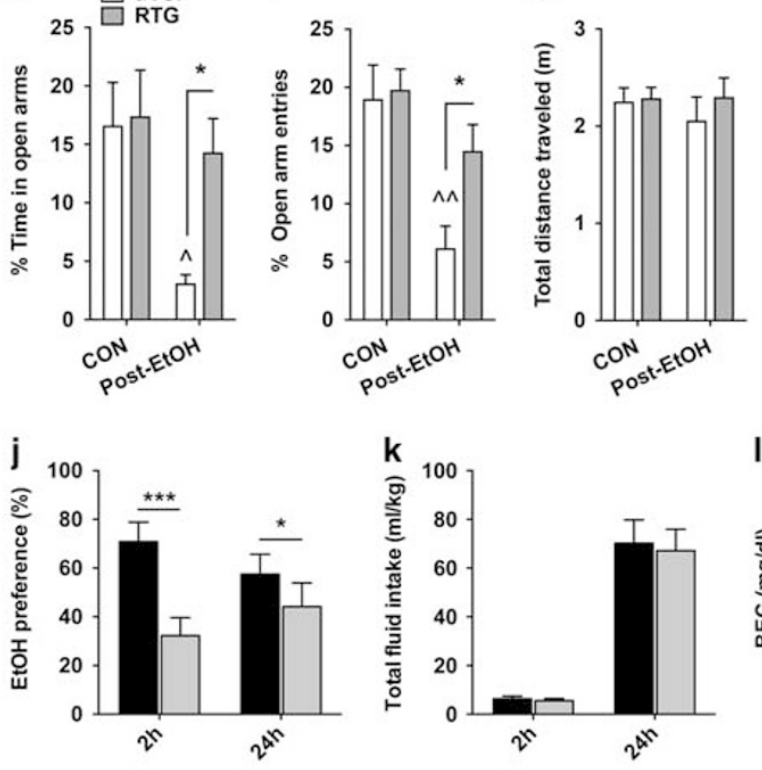

h
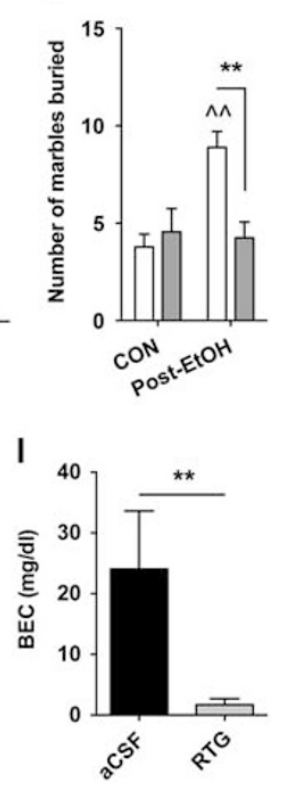

Figure 5 Intra-LHb retigabine reduces anxiety-like behaviors and voluntary ethanol intake. (a-b) Current traces recorded in cell-attached mode (a) and pooled data (b) show that retigabine (RTG)-induced reduction of spontaneous firing was blunted in LHb neurons of Post-EtOH rats compared with ethanol naive rats (CON). Two-way ANOVA, $F_{1,83}=13.19, p=0.0005$, Bonferroni's post hoc test: *** $p<0.0$ I between CON and Post-EtOH. ${ }^{\wedge} p<0.000$ I vs baseline. Numbers in the circles represent $n$ values. (c) Schematic of injection sites in LHb: filled and blank circles are respectively locations for ethanol i.p. injected rats and rats undergoing the intermittent access 2-bottle free choice drinking (IA2BC) procedure. (d-h) LHb infusion of retigabine (RTG, I0 ng/side in $200 \mathrm{nl}$ ) rescues the anxiety-like behaviors of Post-EtOH rats in EPM test ( $\mathrm{d}-\mathrm{g}$ ) and MBT (h). (d) Typical traces in EPM test. (e-g), Intra-LHb infusion of retigabine made Post-EtOH rats spent longer time in the open arms (for drug treatment $F_{1,37}=5.216, p=0.0282$, post hoc test: ^ $p<0.05$ vs $C O N$ aCSF, $* p<0.05$ vs Post-EtOH aCSF) and entered the open arm more often (for drug treatment $F_{1,37}=4.254, p=0.0462$, post hoc test: ^^ $<0.01$ vs CON aCSF, ${ }^{*} p<0.05$ vs Post-EtOH aCSF), but does not significantly change the total distance traveled (for drug treatment, $F_{1,37}=0.42 \mathrm{I} I, p=0.5204$ ), as revealed by two-way ANOVA followed Bonferroni's post hoc test. In the naive (CON) rats, intra-LHb retigabine did not show any significant difference in the time spent in open arms and numbers of open arm entries, and total distance traveled. All post hoc tests: $p>0.05 . \mathrm{N}_{\text {rat }}=10$ (CON aCSF), 9(CON RTG), I I(Post-EtOH aCSF), I I (Post-EtOH RTG). (h) Intra-LHb retigabine also significantly reduced the number of marbles buried in 30-min by Post-EtOH rats. Retigabine did not significantly change the number of marbles buried by CON rats. Two-way ANOVA, for drug treatment $\mathrm{F}_{1,31}=4.58$, $p=0.0403$, Bonferroni's post hoc test: $\wedge \wedge<0.01$ vs CON aCSF, *** $<0.05$ vs Post-EtOH aCSF. N ${ }_{\text {rat }}=9$ (CON aCSF), 9(CON RTG), 9(Post-EtOH aCSF), 8(Post-EtOH RTG). (i-k) Retigabine ( $10 \mathrm{ng} / \mathrm{side}$ in $200 \mathrm{nl}$, intra-LHb) decreased ethanol intake (Paired $t$-test, for $2 \mathrm{~h} t=4.547, p=0.00 \mathrm{I} 4$, for $24 \mathrm{~h}, t=3.778, p=0.0044$, i) and preference (Paired $t$-test, for $2 \mathrm{~h} t=5.930, p=0.0002$, for $24 \mathrm{~h} t=2.484, p=0.0348, \mathrm{j}$ ) without altering total fluid intake at 2 and $24 \mathrm{~h}$ (Paired $t$-test, for $2 \mathrm{~h} t=0.8003$, $p=0.444 \mathrm{I}$, for $24 \mathrm{~h} t=0.5367, p=0.6045, \mathrm{k}$ ) in rats trained to drink in the intermittent access $20 \%$ ethanol two-bottle choice (IA2BC) paradigm. (I) BECs $(\mathrm{mg} / \mathrm{dl})$ after $2 \mathrm{~h}$ of voluntary ethanol consumption in IA2BC procedure. Paired $t$-test: $t=5.93, * * p<0.01$. $\mathrm{N}_{\text {rat }}=10 /$ group.

a dose that changes ethanol intake in rats (McGuier et al, 2015), had significant anxiolytic effects on Post-EtOH rats: these animals spent a significantly longer time in, and entered more frequently into the open arms of the EPM, and reduced the numbers of marbles buried in the MBT, compared to the CON rats (Figure $5 \mathrm{c}-\mathrm{h}$ ).

\section{Activation of LHb M-Channels Reduces Voluntary Alcohol Consumption and Anxiety-like Behaviors of Adult Rats}

Having discovered that LHb M-channels play a critical role in anxiety-related behaviors after ethanol withdrawal, we hypothesized that this mechanism may contribute to the 
drinking behaviors. We therefore trained rats to drink in the intermittent access $20 \%$ ethanol two-bottle free choice paradigm (IA2BC rats). In keeping with previous reports (Li et al, 2011b; Simms et al, 2008), we observed ethanol consumption escalated from $2.5 \pm 0.5 \mathrm{~g} / \mathrm{kg} / 24 \mathrm{~h}$ in the first week to $6.1 \pm 0.9 \mathrm{~g} / \mathrm{kg} / 24 \mathrm{~h}$ in the 8 th week $(n=12$, paired $t$-test, $t=3.08, \quad p=0.015)$. We conducted the following experiments on IA2BC rats that were drinking ethanol for 8-10 weeks when ethanol intake reached a high and stable level. In line with the observation on juvenile rats described above, and on adult rats (Li et al, 2016), the basal firing rate of $\mathrm{LHb}$ neurons in slices obtained at $24 \mathrm{~h}$ withdrawal from IA2BC rats was significantly higher than $\mathrm{CON}$ rats (CON: $4.4 \pm 0.5 \mathrm{~Hz}, n=26$ vs CIEVD: $6.9 \pm 0.5 \mathrm{~Hz}, n=25$, Unpaired $t$-test, $t=3.539, \quad p=0.0009)$. Moreover, XE991-induced acceleration of LHb firing was significantly weaker in slices obtained at $24 \mathrm{~h}$ withdrawal from IA2BC rats compared with CON rats (Supplementary Figure S5A-C).

At $24 \mathrm{~h}$ after withdrawal, these rats showed pronounced anxiety-like behaviors, which were significantly attenuated by LHb infusion of retigabine (Supplementary Figure S5D$G)$. Retigabine infusion also sharply reduced ethanol intake (paired $t$-test, $t=4.547, p=0.0014$ ) and preference (paired $t$-test, $t=5.930, p=0.0002$ ): this effect lasted for $24 \mathrm{~h}$ (paired $t$-test, $t=3.778, p=0.0044$ for EtOH intake and $t=2.484$, $p=0.0348$ for $\mathrm{EtOH}$ preference; Figure $5 \mathrm{i}-\mathrm{k}$ ), but disappeared by $48 \mathrm{~h}$ (not shown). Accordingly, retigabine significantly reduced the blood ethanol level (BEC) measured $2 \mathrm{~h}$ after the access to the ethanol bottles (Paired $t$-test, $t=5.93, p=0.0002$; Figure 51). To determine whether the effect of retigabine was $\mathrm{LHb}$ specific, we infused the same amount of retigabine into the mediodorsal thalamic nuclei or the paraventricular nucleus of thalamus, which are adjacent to the habenula, and failed to detect a significant change in ethanol intake and in anxiety-like behaviors in the EPM (Supplementary Figure S6A-F). Moreover, intra-LHb infusions of retigabine did not significantly alter sucrose intake (Supplementary Figure S6G-H).

\section{DISCUSSION}

We report here evidence of apparent anxiety-like behaviors in rats at $24 \mathrm{~h}$ withdrawal from repeated systemic administration of alcohol. In addition, while the LHb neuronal excitability is increased, the M-current and the expression of the M-channel subunit KCNQ2/3 are reduced. Importantly, chemogenetic inhibition of LHb neurons or intra-LHb administration of the $\mathrm{M}$-channel activator retigabine alleviates the anxiety-like behaviors and reduces alcohol intake. These findings suggest that M-channels in the LHb play a significant role in anxiety-related behaviors after ethanol withdrawal.

In this study, we first described apparent anxiety-like behaviors in rats withdrawn from in vivo systemic ethanol administration, as shown by the reduced time spent in the center of an open field, and in the open arms of a maze, as well as the increased number of buried marbles. Since the total distance traveled was not different, ethanol withdrawal did not noticeably affect locomotor activity. These anxiety-like behaviors were observed in rats $24 \mathrm{~h}$ after the end of systemic administration of ethanol (passive or voluntary), and in both juvenile and adult rats. Our data are thus consistent with previous reports of the anxiogenic effects of ethanol on rodents (Baldwin et al, 1991; Knapp et al, 2005). Notably, the MBT measures not only anxiety value (Njung'e and Handley, 1991; Zhao-Shea et al, 2015), but also obsessive compulsive disorder (OCD) (Albelda and Joel, 2012). As OCD commonly occurs alongside drug and alcohol addiction (De Ridder et al, 2016; Karg et al, 2012), our MBT data thus suggest an important role of the LHb in OCD.

Although the LHb has been linked with anxiety-related behaviors (Chan et al, 2016; Dolzani et al, 2016; Pobbe and Zangrossi, 2008; Shelton et al, 2016), its role in the context of ethanol withdrawal was unknown. We found that LHb neurons in ethanol-withdrawn (Post-EtOH) rats had a significantly higher spontaneous firing rate (Li et al, 2016) and excitability, suggesting a possible contribution of LHb hyperactivity to the increased anxiety levels. This possibility was supported by our data showing that selective inhibition of $\mathrm{LHb}$ neurons by chemogenetic or pharmacological approaches mitigated elevated anxiety-like phenotypes associated with ethanol withdrawal, and that selective activation of LHb neurons can induce anxiety-like phenotypes. Searching for the underlying cellular and molecular mechanisms, we found that the LHb neurons of Post-EtOH rats had a smaller mAHP, compared to CON rats, and that the M-channel blocker XE991 significantly increased the firing rate of $\mathrm{LHb}$ neurons in $\mathrm{CON}$ but not Post-EtOH rats, suggesting that $\mathrm{M}$-channel dysregulation may contribute to the observed increased excitability of LHb neurons in PostEtOH rats. This possibility was supported by the observation that in the Post-EtOH rats, both M-current and KCNQ2/3 expression was reduced, and that the M-channel activator retigabine reduced $\mathrm{LHb}$ neuron firing. These data support a link between LHb activity and anxiety-like behaviors.

Previous studies have associated M-channels with ethanol intake (Knapp et al, 2014; McGuier et al, 2015), and have proposed M-channels as a target of ethanol's actions on neuronal function. These studies showed that acute ethanol inhibits M-currents in human embryonic kidney cells expressing KCNQ2/3 (Cavaliere et al, 2012), in rat VTA dopamine neurons (Koyama et al, 2007), and in rat hippocampal pyramidal neurons (Moore et al, 1990); and chronic ethanol exposure downregulates KCNQ2 expression in synaptoneurosomes of mice (Most et al, 2015). In addition, M-channels regulate tolerance and memory impairments induced by acute ethanol in Drosophila (Cavaliere et al, 2012). Consistent with these findings, we showed that intra$\mathrm{LHb}$ infused retigabine attenuates anxiety levels and reduces ethanol intake and preference. Our data of the anxiolytic effect of retigabine are consistent with previous reports (Hansen et al, 2008; Korsgaard et al, 2005). Thus, retigabine is a potential therapeutic option for alcoholics. This possibility is supported by recent reports that either systemic administration, or infusion of retigabine into the nucleus accumbens significantly reduces ethanol intake in rats (Knapp et al, 2014; McGuier et al, 2015). Notably, since retigabine has been approved by the US food and drug administration for epilepsy treatment, clinical trials could be started relatively quickly. However, caution must be exercised since M-channels have been associated with cognitive functions (Millichap and Cooper, 2012). 
The observed reduction of M-channel protein expression during ethanol withdrawal may be caused by changes in the activity of transcriptional factors that suppress the corresponding gene expression. The transcription factor SP1 and transcription repressor REST (repressor element 1-silencing transcription factor) have been identified as common mechanisms of regulating KCNQ2 and KCNQ3 expression (Mucha et al, 2010; Rose et al, 2011). Indeed, the activity of the transcription factor that suppresses KCNQ2 expression is enhanced after ethanol exposure. Ethanol increases REST expression levels or enhances the REST binding activity to its binding site, RE-1, in an ethanol concentration-dependent manner (Cai et al, 2011; Ishii et al, 2008; Tateno et al, 2006). Transcriptional regulation via transcription repressors, including REST, has been suggested as a therapeutic option for anxiety and depression because REST activity may be a common mechanism underlying the pathophysiology of anxiety and depression (Albert and Fiori, 2014). This may also explain why enhanced LHb neuronal excitability contributes to depression ( $\mathrm{Li}$ et al, 2011a, 2013). Given the role of LHb in depression-linked behaviors (Lecca et al, 2014; Li et al, 2013) and the connection between anxiety and depression (Pini et al, 1997), it will be interesting to investigate the role of $\mathrm{LHb} \mathrm{M}$-channel in depression.

Although we did not identify the cell type we recorded from in the current study, they most likely are glutamatergic since $95 \%$ of LHb neurons are glutamatergic (Meye et al, 2013; Suzuki et al, 2012; Weiss and Veh, 2011). As mentioned, the $\mathrm{LHb}$ consists of the lateral and the medial parts, each having different connectivity. Whereas the lateral part projects mainly to DA neurons in the VTA and substantial nigra, indirectly via the rostromedial tegmental nucleus (Aizawa et al, 2013; Proulx et al, 2014), the medial part projects mainly to the serotonergic system (dorsal and median raphe nuclei, DR and MR) (Proulx et al, 2014). The $\mathrm{DR}$ is the largest cluster of ascending serotonergic projections in the rat brain (Ferron et al, 1982), which participates in mediating anxiety- and depression-related behaviors (Dolzani et al, 2016; Graeff et al, 1996; Teissier et al, 2015). Conversely, the VTA also has been known as a pivotal area to modulate anxiety and reward motivation when animals are exposed to a known anxiety-causing environment (Tovote et al, 2015). The functional significance of the various sub-connections from the LHb during ethanol withdrawal remains unclear. Future studies thus are needed to clarify which circuit (the LHb-DR/MR or the LHb-VTA) is more heavily affected by ethanol exposure and withdrawal.

Interestingly, a previous rat study found increased alcohol consumption after LHb lesion (Haack et al, 2014), in contrast to the reduced alcohol intake following retigabine inhibition of the LHb observed in the current study. The mechanisms underlying the apparent disparity are unclear. However, the conditions of these two experiments are very different. One major difference is the time when retigabine or the lesion was applied. In Haack's study, the LHb was lesioned 1 week before the animal was trained to drink alcohol. LHb lesions increased the rate of escalation of intake, leading to higher consumption levels. By contrast, in our current study, retigabine was applied to the $\mathrm{LHb}$ of rats that have been drinking ethanol for two months, when the animals probably became dependent on alcohol (Fu et al, 2015; Li et al, 2011b). Our data of LHb inhibition leading to reduced drinking is in line with our recent finding that high frequency deep brain stimulation, which inhibits the $\mathrm{LHb}$, leads to reduced ethanol drinking ( $\mathrm{Li}$ et al, 2016). One limitation of the current study is that although our data support a link between increased LHb activity and anxiety-like behaviors, direct evidence for anxiety contributing to increased alcohol intake is still lacking.

In summary, we provide here several lines of new information about the role of LHb M-channels in the anxiogenic effect of ethanol withdrawal. The increased anxiety-like behaviors of ethanol-withdrawn rats were paralleled by increased excitability of LHb neurons, and reduced M-current and M-channel expression. Activation of M-channels attenuates both ethanol drinking and anxietylike behaviors. These findings thus identify downregulation of M-channels in LHb as an anxiogenic mechanism, and suggest an important role of the M-channels in ethanolinduced neuronal adaptation. Thus, M-channels could be a promising therapeutic target for alcoholics.

\section{FUNDING AND DISCLOSURE}

This work was funded by NIH grants AA021657 and AA022292, and a grant from New Jersey Health foundation. Clozapine-n-oxide (CNO) was from NIDA Drug supply program (NIH, Bethesda, MD). The authors declare no conflict of interest.

\section{ACKNOWLEDGMENTS}

We thank Dr Rose Paulose for her careful reading of the manuscript.

\section{AUTHOR CONTRIBUTIONS}

SK conceived and designed all molecular and behavioral experiments. SK, JL, and WZ performed stereotaxic surgery and behavioral experiments. SK performed electrophysiology. SK and JL performed western blotting. SK and RF performed immunofluorescence. SK and JY performed statistical analysis, prepared figures and wrote the manuscript. All authors contributed to reviewing and editing the manuscript.

\section{REFERENCES}

Aizawa H, Cui W, Tanaka K, Okamoto H (2013). Hyperactivation of the habenula as a link between depression and sleep disturbance. Front Hum Neurosci 7: 826.

Albelda N, Joel D (2012). Current animal models of obsessive compulsive disorder: an update. Neuroscience 211: 83-106.

Albert PR, Fiori LM (2014). Transcriptional dys-regulation in anxiety and major depression: 5-HT1A gene promoter architecture as a therapeutic opportunity. Curr Pharm Des 20: 3738-3750.

Alexander GM, Rogan SC, Abbas AI, Armbruster BN, Pei Y, Allen JA et al (2009). Remote control of neuronal activity in transgenic mice expressing evolved G protein-coupled receptors. Neuron 63: 27-39.

Armbruster BN, Li X, Pausch MH, Herlitze S, Roth BL (2007). Evolving the lock to fit the key to create a family of G proteincoupled receptors potently activated by an inert ligand. Proc Natl Acad Sci USA 104: 5163-5168. 
Baldwin HA, Rassnick S, Rivier J, Koob GF, Britton KT (1991). CRF antagonist reverses the 'anxiogenic' response to ethanol withdrawal in the rat. Psychopharmacology 103: 227-232.

Baldwin PR, Alanis R, Salas R (2011). The role of the habenula in nicotine addiction. J Addict Res Ther Suppl 1: 002.

Cai L, Bian M, Liu M, Sheng Z, Suo H, Wang Z et al (2011). Ethanol-induced neurodegeneration in NRSF/REST neuronal conditional knockout mice. Neuroscience 181: 196-205.

Carnicella S, Amamoto R, Ron D (2009). Excessive alcohol consumption is blocked by glial cell line-derived neurotrophic factor. Alcohol 43: 35-43.

Castro PA, Cooper EC, Lowenstein DH, Baraban SC (2001). Hippocampal heterotopia lack functional Kv4.2 potassium channels in the methylazoxymethanol model of cortical malformations and epilepsy. J Neurosci 21: 6626-6634.

Cavaliere S, Gillespie JM, Hodge JJ (2012). KCNQ channels show conserved ethanol block and function in ethanol behaviour. PLoS ONE 7: e50279.

Chan J, Ni Y, Zhang P, Chen Y, Zhang J (2016). D1-like dopamine receptor dysfunction in the lateral habenula nucleus increased anxiety-like behavior in rat. Neuroscience 340: 542-550.

De Ridder D, Leong SL, Manning P, Vanneste S, Glue P (2016). Anterior cingulate implant for obsessive-compulsive disorder. World Neurosurg 97: 754.e7-754.e16.

Delmas P, Brown DA (2005). Pathways modulating neural KCNQ/ M (Kv7) potassium channels. Nat Rev Neurosci 6: 850-862.

Dolzani SD, Baratta MV, Amat J, Agster KL, Saddoris MP, Watkins LR et al (2016). Activation of a Habenulo-Raphe circuit is critical for the behavioral and neurochemical consequences of uncontrollable stress in the male rat. eNeuro 3: ENEURO.022916.2016.

Driessen M, Meier S, Hill A, Wetterling T, Lange W, Junghanns K (2001). The course of anxiety, depression and drinking behaviours after completed detoxification in alcoholics with and without comorbid anxiety and depressive disorders. Alcohol Alcohol 36: 249-255.

Ferron A, Descarries L, Reader TA (1982). Altered neuronal responsiveness to biogenic amines in rat cerebral cortex after serotonin denervation or depletion. Brain Res 231: 93-108.

Fu R, Gregor D, Peng Z, Li J, Bekker A, Ye J (2015). Chronic intermittent voluntary alcohol drinking induces hyperalgesia in Sprague-Dawley rats. Int J Physiol Pathophysiol Pharmacol 7: 136-144.

Gibula-Bruzda E, Marszalek-Grabska M, Witkowska E, Izdebski J, Kotlinska JH (2015). Enkephalin analog, cyclo[N(epsilon),N (beta)-carbonyl-D-Lys(2),Dap(5)] enkephalinamide (cUENK6), inhibits the ethanol withdrawal-induced anxiety-like behavior in rats. Alcohol 49: 229-236.

Gilpin NW, Herman MA, Roberto M (2015). The central amygdala as an integrative hub for anxiety and alcohol use disorders. Biol Psychiatry 77: 859-869.

Graeff FG, Guimaraes FS, De Andrade TG, Deakin JF (1996). Role of 5-HT in stress, anxiety, and depression. Pharmacol Biochem Behav 54: 129-141.

Gu N, Vervaeke K, Hu H, Storm JF (2005). Kv7/KCNQ/M and $\mathrm{HCN} / \mathrm{h}$, but not $\mathrm{KCa} 2 / \mathrm{SK}$ channels, contribute to the somatic medium after-hyperpolarization and excitability control in CA1 hippocampal pyramidal cells. J Physiol 566(Pt 3): 689-715.

Haack AK, Sheth C, Schwager AL, Sinclair MS, Tandon S, Taha SA (2014). Lesions of the lateral habenula increase voluntary ethanol consumption and operant self-administration, block yohimbineinduced reinstatement of ethanol seeking, and attenuate ethanolinduced conditioned taste aversion. PLoS ONE 9: e92701.

Halter JA, Carp JS, Wolpaw JR (1995). Operantly conditioned motoneuron plasticity: possible role of sodium channels. J Neurophysiol 73: 867-871.
Hansen HH, Waroux O, Seutin V, Jentsch TJ, Aznar S, Mikkelsen JD (2008). Kv7 channels: interaction with dopaminergic and serotonergic neurotransmission in the CNS. J Physiol 586: $1823-1832$.

Hikosaka O (2010). The habenula: from stress evasion to valuebased decision-making. Nat Rev Neurosci 11: 503-513.

Ishii T, Hashimoto E, Ukai W, Tateno M, Yoshinaga T, Saito S et al (2008). Lithium-induced suppression of transcription repressor NRSF/REST: effects on the dysfunction of neuronal differentiation by ethanol. Eur J Pharmacol 593: 36-43.

Kang S, Xu M, Cooper EC, Hoshi N (2014). Channel-anchored protein kinase CK2 and protein phosphatase 1 reciprocally regulate KCNQ2-containing M-channels via phosphorylation of calmodulin. J Biol Chem 289: 11536-11544.

Karg RS, Bose J, Batts KR, Forman-Hoffman VL, Liao D, Hirsch E et al (2012). Past year mental disorders among adults in the United States: results from the 2008-2012 Mental Health Surveillance Study. In: CBHSQ Data Review, Rockville, MD, pp 1-19.

Klinger F, Gould G, Boehm S, Shapiro MS (2011). Distribution of M-channel subunits KCNQ2 and KCNQ3 in rat hippocampus. Neuroimage 58: 761-769.

Knapp CM, O'Malley M, Datta S, Ciraulo DA (2014). The Kv7 potassium channel activator retigabine decreases alcohol consumption in rats. Am J Drug Alcohol Abuse 40: 244-250.

Knapp DJ, Overstreet DH, Breese GR (2005). Modulation of ethanol withdrawal-induced anxiety-like behavior during later withdrawals by treatment of early withdrawals with benzodiazepine/ gamma-aminobutyric acid ligands. Alcohol Clin Exp Res 29: 553-563.

Korsgaard MP, Hartz BP, Brown WD, Ahring PK, Strobaek D, Mirza NR (2005). Anxiolytic effects of Maxipost (BMS-204352) and retigabine via activation of neuronal Kv7 channels. J Pharmacol Exp Ther 314: 282-292.

Kourrich S, Calu DJ, Bonci A (2015). Intrinsic plasticity: an emerging player in addiction. Nat Rev Neurosci 16: 173-184.

Koyama S, Brodie MS, Appel SB (2007). Ethanol inhibition of $\mathrm{m}$-current and ethanol-induced direct excitation of ventral tegmental area dopamine neurons. J Neurophysiol 97: 1977-1985.

Lecca S, Meye FJ, Mameli M (2014). The lateral habenula in addiction and depression: an anatomical, synaptic and behavioral overview. Eur J Neurosci 39: 1170-1178.

Li B, Piriz J, Mirrione M, Chung C, Proulx CD, Schulz D et al (2011a). Synaptic potentiation onto habenula neurons in the learned helplessness model of depression. Nature 470: 535-539.

Li J, Bian W, Dave V, Ye JH (2011b). Blockade of GABA(A) receptors in the paraventricular nucleus of the hypothalamus attenuates voluntary ethanol intake and activates the hypothalamicpituitary-adrenocortical axis. Addict Biol 16: 600-614.

Li J, Zuo W, Fu R, Xie G, Kaur A, Bekker A et al (2016). High frequency electrical stimulation of lateral habenula reduces voluntary ethanol consumption in rats. Int J Neuropsychopharmacol 19: pyw050.

Li K, Zhou T, Liao L, Yang Z, Wong C, Henn F et al (2013). betaCaMKII in lateral habenula mediates core symptoms of depression. Science 341: 1016-1020.

McBride WJ (2002). Central nucleus of the amygdala and the effects of alcohol and alcohol-drinking behavior in rodents. Pharmacol Biochem Behav 71: 509-515.

McGuier NS, Griffin WC 3rd, Gass JT, Padula AE, Chesler EJ, Mulholland PJ (2015). Kv7 channels in the nucleus accumbens are altered by chronic drinking and are targets for reducing alcohol consumption. Addict Biol 21: 1097-1112.

Meye FJ, Lecca S, Valentinova K, Mameli M (2013). Synaptic and cellular profile of neurons in the lateral habenula. Front Hum Neurosci 7: 860. 
Millichap JJ, Cooper EC (2012). KCNQ2 potassium channel epileptic encephalopathy syndrome: divorce of an electromechanical couple? Epilepsy Curr 12: 150-152.

Misonou H (2010). Homeostatic regulation of neuronal excitability by $\mathrm{K}(+)$ channels in normal and diseased brains. Neuroscientist 16: 51-64.

Moore SD, Madamba SG, Siggins GR (1990). Ethanol diminishes a voltage-dependent $\mathrm{K}+$ current, the $\mathrm{M}$-current, in CA1 hippocampal pyramidal neurons in vitro. Brain Res 516: 222-228.

Most D, Ferguson L, Blednov Y, Mayfield RD, Harris RA (2015). The synaptoneurosome transcriptome: a model for profiling the emolecular effects of alcohol. Pharmacogenomics $J$ 15: $177-188$.

Mucha M, Ooi L, Linley JE, Mordaka P, Dalle C, Robertson B et al (2010). Transcriptional control of KCNQ channel genes and the regulation of neuronal excitability. I Neurosci 30: $13235-13245$.

Njung'e K, Handley SL (1991). Evaluation of marble-burying behavior as a model of anxiety. Pharmacol Biochem Behav 38: 63-67.

Pandey SC, Roy A, Zhang H (2003). The decreased phosphorylation of cyclic adenosine monophosphate (cAMP) response element binding (CREB) protein in the central amygdala acts as a molecular substrate for anxiety related to ethanol withdrawal in rats. Alcohol Clin Exp Res 27: 396-409.

Pandey SC, Zhang H, Roy A, Misra K (2006). Central and medial amygdaloid brain-derived neurotrophic factor signaling plays a critical role in alcohol-drinking and anxiety-like behaviors. J Neurosci 26: 8320-8331.

Pedarzani P, Kulik A, Muller M, Ballanyi K, Stocker M (2000). Molecular determinants of $\mathrm{Ca} 2+$-dependent $\mathrm{K}+$ channel function in rat dorsal vagal neurones. J Physiol 527(Pt 2): 283-290.

Pini S, Cassano GB, Simonini E, Savino M, Russo A, Montgomery SA (1997). Prevalence of anxiety disorders comorbidity in bipolar depression, unipolar depression and dysthymia. J Affect Disord 42: 145-153.

Pobbe RL, Zangrossi H Jr (2008). Involvement of the lateral habenula in the regulation of generalized anxiety- and panicrelated defensive responses in rats. Life Sci 82: 1256-1261.

Proulx CD, Hikosaka O, Malinow R (2014). Reward processing by the lateral habenula in normal and depressive behaviors. Nat Neurosci 17: 1146-1152.

Rose K, Ooi L, Dalle C, Robertson B, Wood IC, Gamper N (2011). Transcriptional repression of the $\mathrm{M}$ channel subunit Kv7.2 in chronic nerve injury. Pain 152: 742-754.

Sena LM, Bueno C, Pobbe RL, Andrade TG, Zangrossi H Jr, Viana MB (2003). The dorsal raphe nucleus exerts opposed control on generalized anxiety and panic-related defensive responses in rats. Behav Brain Res 142: 125-133.

Shah M, Mistry M, Marsh SJ, Brown DA, Delmas P (2002). Molecular correlates of the M-current in cultured rat hippocampal neurons. J Physiol 544(Pt 1): 29-37.

Shelton K, Bogyo K, Schick T, Ettenberg A (2016). Pharmacological modulation of lateral habenular dopamine D2 receptors alters the anxiogenic response to cocaine in a runway model of drug selfadministration. Behav Brain Res 310: 42-50.

Simms JA, Steensland P, Medina B, Abernathy KE, Chandler LJ, Wise $\mathrm{R}$ et al (2008). Intermittent access to $20 \%$ ethanol induces high ethanol consumption in Long-Evans and Wistar rats. Alcohol Clin Exp Res 32: 1816-1823.
Sinha R (2001). How does stress increase risk of drug abuse and relapse? Psychopharmacology 158: 343-359.

Smith KS, Bucci DJ, Luikart BW, Mahler SV (2016). DREADDS: use and application in behavioral neuroscience. Behav Neurosci 130: 137-155.

Spiacci A Jr, Coimbra NC, Zangrossi H Jr (2012). Differential involvement of dorsal raphe subnuclei in the regulation of anxietyand panic-related defensive behaviors. Neuroscience 227: 350-360.

Suzuki A, Ishida Y, Aizawa S, Sakata I, Tsutsui C, Mondal A et al (2012). Molecular identification of GHS-R and GPR38 in Suncus murinus. Peptides 36: 29-38.

Tateno M, Ukai W, Hashimoto E, Ikeda H, Saito T (2006). Implication of increased NRSF/REST binding activity in the mechanism of ethanol inhibition of neuronal differentiation. J Neural Transm 113: 283-293.

Teissier A, Chemiakine A, Inbar B, Bagchi S, Ray RS, Palmiter RD et al (2015). Activity of Raphe serotonergic neurons controls emotional behaviors. Cell Rep 13: 1965-1976.

Tovote P, Fadok JP, Luthi A (2015). Neuronal circuits for fear and anxiety. Nat Rev Neurosci 16: 317-331.

Trimmer JS (2015). Subcellular localization of $\mathrm{K}+$ channels in mammalian brain neurons: remarkable precision in the midst of extraordinary complexity. Neuron 85: 238-256.

Vervaeke K, Gu N, Agdestein C, Hu H, Storm JF (2006). Kv7/ KCNQ/M-channels in rat glutamatergic hippocampal axons and their role in regulation of excitability and transmitter release. J Physiol 576(Pt 1): 235-256.

Vielhaber S, Feistner H, Weis J, Kreuder J, Sailer M, Schroder JM et al (2004). Primary carnitine deficiency: adult onset lipid storage myopathy with a mild clinical course. J Clin Neurosci 11: 919-924.

Wang HS, Pan Z, Shi W, Brown BS, Wymore RS, Cohen IS et al (1998). KCNQ2 and KCNQ3 potassium channel subunits: molecular correlates of the M-channel. Science 282: 1890-1893.

Waxman SG, Zamponi GW (2014). Regulating excitability of peripheral afferents: emerging ion channel targets. Nat Neurosci 17: $153-163$.

Weiss T, Veh RW (2011). Morphological and electrophysiological characteristics of neurons within identified subnuclei of the lateral habenula in rat brain slices. Neuroscience 172: 74-93.

Wise RA (1973). Voluntary ethanol intake in rats following exposure to ethanol on various schedules. Psychopharmacologia 29: 203-210.

Wright S, Sternberg H, Bjornskov EK, Stephenson DT, Kushner PD (1990). Family of human neuronal external surface epitopes defined by Torpedo monoclonal antibodies. J Neurosci Res 25: 486-502.

Zhang XF, Hu XT, White FJ (1998). Whole-cell plasticity in cocaine withdrawal: reduced sodium currents in nucleus accumbens neurons. J Neurosci 18: 488-498.

Zhao H, Zhang BL, Yang SJ, Rusak B (2015). The role of lateral habenula-dorsal raphe nucleus circuits in higher brain functions and psychiatric illness. Behav Brain Res 277: 89-98.

Zhao-Shea R, DeGroot SR, Liu L, Vallaster M, Pang X, Su Q et al (2015). Increased CRF signalling in a ventral tegmental areainterpeduncular nucleus-medial habenula circuit induces anxiety during nicotine withdrawal. Nat Commun 6: 6770.

Zuo W, Fu R, Hopf FW, Xie G, Krnjevic K, Li J et al (2015). Ethanol drives aversive conditioning through dopamine 1 receptor and glutamate receptor-mediated activation of lateral habenula neurons. Addict Biol 22: 103-116.

Supplementary Information accompanies the paper on the Neuropsychopharmacology website (http://www.nature.com/npp) 